\title{
Canonical and path-integral quantization of string cosmology models
}

\author{
Marco Cavaglià† and Carlo Ungarellił \\ Max-Planck-Institut für Gravitationsphysik, Albert-Einstein-Institut, Schlaatzweg 1, D-14473 \\ Potsdam, Germany
}

Received 11 December 1998

\begin{abstract}
We discuss the quantization of a class of string cosmology models that are characterized by scale factor duality invariance. We compute the amplitudes for the full set of classically allowed and forbidden transitions by applying the reduce phase space and the path-integral methods. We show that these approaches are consistent. The path-integral calculation clarifies the meaning of the instanton-like behaviour of the transition amplitudes that was first pointed out in previous investigations.
\end{abstract}

PACS numbers: 0460K, 9880C, 9880H

\section{Introduction}

String theory, thanks to its duality symmetries, provides a cosmological scenario [1-3] in which the Universe starts from the perturbative vacuum of (super)string theory and evolves in a 'pre-big bang' (PRBB) phase [1,2]§ characterized by an accelerated growth of the curvature and of the string coupling.

One of the main problems of string cosmology is the understanding of the mechanism responsible for the transition ('graceful exit') from the inflationary PRBB phase to the deflationary 'post-big bang' phase (POBB) with decreasing curvature that is typical of the standard cosmological scenario. Necessarily, the graceful exit involves a high-curvature, strong-coupling, regime where higher derivatives [4] and string loop terms must be taken into account. In [5] it has been shown that for any choice of the (local) dilaton potential, no cosmological solutions that connect smoothly the PRBB and POBB phases exist. Consequently, at the classical level higher-order corrections cannot be 'simulated' by any realistic dilaton potential.

At the quantum level the dilaton potential may induce the transition from the PRBB phase to the POBB phase. In this context, using the standard Dirac method of quantization based on the Wheeler-De Witt equation [6] a number of minisuperspace models have been investigated in the literature [7-9]. The result of these investigations is a finite, non-zero, transition probability $\mathrm{PRBB} \rightarrow \mathrm{POBB}$ with a typical 'instanton-like' dependence $\left(\sim \exp \left\{-1 / g^{2}\right\}\right)$ on the string coupling constant $[7,8]$.

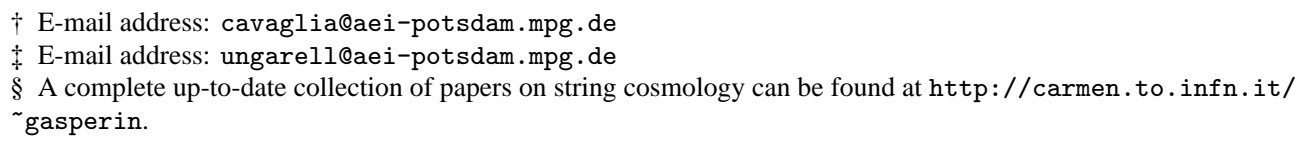


The aim of this paper is to present a refined analysis of the quantization of string cosmological models. To this end we reconsider the minisuperspace models that have been previously investigated in [7-9]. We have several motivations for doing this.

First of all, these systems are invariant under reparametrization of time. So their quantization requires a careful discussion of the subtleties that are typical of the quantization of gauge-invariant systems (e.g. gauge fixing) $[10,11]$. Furthermore, we want to investigate the graceful exit in string cosmology using different techniques of quantization and illustrate a consistent approach to the problem that can be successfully applied to a large class of models.

We deal with a class of string-inspired models-see (2.8) and (2.9) below-that are exactly integrable and we apply the standard techniques for the canonical quantization of constrained systems [12-14]. Using the reduced-phase space formalism we determine the positive norm Hilbert space of states. We construct the PRBB and POBB wavefunctions that are normalized with respect to the inner product of the Hilbert space. These wavefunctions are then used to compute transition amplitudes. Further, we compute the (semiclassical) transition amplitude PRBB $\rightarrow$ POBB by the path-integral approach. The result agrees with the semiclassical limit of the transition amplitude that has been obtained in the reduced-phase space approach and makes clear the instanton-like structure pointed out in [7,8]. Let us stress that our investigation is important for at least two reasons: first, the model that we are discussing is (to our knowledge) the only known example of a minisuperspace model where exact transition probabilities between two classically disconnected backgrounds have been calculated. Secondly, our analysis completes the previous investigations of [7-9] and allows for a systematic discussion of both classically allowed and classically forbidden transitions.

The outline of the paper is as follows. Section 2 is devoted to the classical theory. We derive the solutions of the equations of motion and discuss the classical behaviour of the PRBB and POBB branches. In section 3 we quantize the model. This task is completed using first the canonical approach and then the path-integral formalism. Eventually, we state our conclusions in section 4 .

\section{Classical theory}

We consider the string-inspired model in $d+1$ dimensions described by the action (we assume that only the metric and the dilaton contribute non-trivially to the background)

$$
S=\frac{1}{2 \lambda_{s}^{d-1}} \int \mathrm{d}^{d+1} x \sqrt{|g|} \mathrm{e}^{-\phi}\left(R+\partial_{\mu} \phi \partial^{\mu} \phi-V\left(g_{\mu \nu}, \phi\right)\right),
$$

where $\phi$ is the dilaton field, $\lambda_{s}=\left(\alpha^{\prime}\right)^{1 / 2}$ is the fundamental string length parameter and $V\left(g_{\mu \nu}, \phi\right)$ is a potential term. When the latter is absent, equation (2.1) coincides with the treelevel, lowest order in $\alpha$ ', string effective action [15] defined in the 'string frame', where the metric $g_{\mu \nu}$ coincides with the $\sigma$-model background metric that couples directly to the strings.

We deal with isotropic, spatially flat, cosmological backgrounds parametrized by

$g_{\mu \nu}=\operatorname{diag}\left(-N^{2}(t), a^{2}(t) \delta_{i j}\right), \quad a=\exp [\Theta(t) / \sqrt{d}], \quad \phi=\phi(t)$,

where $i, j=1, \ldots, d$. We also assume that the spatial sections have finite volume. For this class of backgrounds the action (2.1) reads

$$
S=\int \mathrm{d} t \mathcal{L}, \quad \mathcal{L}=\frac{\lambda_{s}}{2}\left(\frac{1}{\mu}\left(\dot{\Theta}^{2}-\dot{\Phi}^{2}\right)-\mu \mathrm{e}^{-2 \Phi} V(\Theta, \Phi)\right),
$$

where dots represent differentiation with respect to cosmic time $t, \mu(t)=N(t) \mathrm{e}^{\Phi}$, and $\Phi$ is the 'shifted' dilaton field

$$
\Phi=\phi-\log \int \mathrm{d}^{d} x / \lambda_{s}^{d}-\sqrt{d} \Theta .
$$


(In (2.3) we have neglected surface terms that are inessential for our purposes.) In this paper we restrict attention to models with a potential term depending on the shifted dilaton $\Phi$ only. In this case (2.3) is invariant under scale factor duality transformations $[1,16]$

$$
\begin{aligned}
& \Phi \rightarrow \Phi \quad \text { or } \quad \phi \rightarrow \phi-2 d \log a, \\
& \Theta \rightarrow-\Theta \quad \text { or } \quad a \rightarrow \frac{1}{a} .
\end{aligned}
$$

Let us introduce the conjugate momenta to $\Theta$ and $\Phi$ by the Legendre transformation

$$
\Pi_{\Theta}=\frac{\lambda_{s}}{\mu} \dot{\Theta}, \quad \Pi_{\Phi}=-\frac{\lambda_{s}}{\mu} \dot{\Phi} .
$$

Equation (2.3) can be cast in the canonical form

$$
S=\int \mathrm{d} t\left[\dot{\Theta} \Pi_{\Theta}+\dot{\Phi} \Pi_{\Phi}-\mathcal{H}\right],
$$

where

$$
\mathcal{H}=\mu(t) H, \quad H=\frac{1}{2 \lambda_{s}}\left(\Pi_{\Theta}^{2}-\Pi_{\Phi}^{2}+\lambda_{s}^{2} V(\Phi) \mathrm{e}^{-2 \Phi}\right) .
$$

In the canonical formalism $\mu$ plays the role of a non-dynamical variable that enforces the constraint $H=0$. As we expect for a time-reparametrization-invariant system, the total Hamiltonian $\mathcal{H}$ is proportional to the constraint $[13,14]$. The equations of motion are

$$
\begin{array}{ll}
\frac{\mathrm{d} \Theta}{\mathrm{d} \tau}=\frac{\Pi_{\Theta}}{\lambda_{s}}, & \frac{\mathrm{d} \Phi}{\mathrm{d} \tau}=-\frac{\Pi_{\Phi}}{\lambda_{s}}, \\
\frac{\mathrm{d} \Pi_{\Theta}}{\mathrm{d} \tau}=0, & \frac{\mathrm{d} \Pi_{\Phi}}{\mathrm{d} \tau}=\frac{\lambda_{s}}{2}\left(2 V-\frac{\mathrm{d} V}{\mathrm{~d} \Phi}\right) \mathrm{e}^{-2 \Phi},
\end{array}
$$

where $\tau(t)$ is

$$
\tau(t)=\int^{t} \mathrm{~d} s \mu(s)
$$

The gauge parameter $\tau(t)$ is related to the synchronous-gauge time $t^{(s g)}(N=1)$ by the relation

$$
t^{(s g)}(\tau)=\int^{\tau} \mathrm{d} s \mathrm{e}^{-\Phi} .
$$

We consider potentials of the form

$$
V(\Phi)=\lambda \mathrm{e}^{-2 \Phi(q-1)},
$$

where $\lambda>0$ is a dimension-two quantity (in natural units) and $q$ is a dimensionless parameter. (This class of potentials has been first discussed in [9].) For $q \neq 0$ the explicit solution of the equations of motion (2.10) and (2.11) is

$$
\begin{aligned}
& \Theta=\Theta_{0}+\frac{k}{\lambda_{s}}\left(\tau-\tau_{0}\right), \quad \quad \mathrm{e}^{\Phi}=\left[\frac{\sqrt{\lambda} \lambda_{s}}{|k|} \sinh \left(\frac{|k q|}{\lambda_{s}}\left|\tau-\tau_{0}\right|\right)\right]^{1 / q}, \\
& \Pi_{\Theta}=k, \quad \Pi_{\Phi}=-k \operatorname{coth}\left[\frac{k q}{\lambda_{s}}\left(\tau-\tau_{0}\right)\right] .
\end{aligned}
$$

(The case $q=0$ corresponds - modulo a redefinition of $k$ - to the 'vacuum' solutions discussed in [1-3].) Let us determine which values of $q$ do allow for the existence of an inflationary expanding PRBB branch and a decelerating POBB branch. According to the general analysis of $[1,2]$, the expanding PRBB and POBB branches are defined by

$$
\begin{array}{llll}
\text { PRBB: } & \boldsymbol{H}>0, & \dot{\boldsymbol{H}}>0, & \dot{\Phi}>0, \\
\text { POBB: } & \boldsymbol{H}>0, & \dot{\boldsymbol{H}}<0, & \dot{\Phi}<0,
\end{array}
$$


where

$$
\begin{aligned}
& \boldsymbol{H}=\frac{\mathrm{d}}{\mathrm{d} t^{(s g)}}(\log a)=\frac{1}{\sqrt{d}} \frac{k}{\lambda_{s}} \mathrm{e}^{\Phi}, \\
& \dot{H}=\frac{\mathrm{d}}{\mathrm{d} t^{(s g)}} \boldsymbol{H}=\frac{1}{\sqrt{d}} \frac{k}{2 \lambda_{s}} \frac{\mathrm{d}}{\mathrm{d} \tau}\left(\mathrm{e}^{2 \Phi}\right), \\
& \dot{\Phi}=\frac{\mathrm{d}}{\mathrm{d} t^{(s g)}} \Phi=\frac{\mathrm{d}}{\mathrm{d} \tau}\left(\mathrm{e}^{\Phi}\right) .
\end{aligned}
$$

From (2.18) it is straightforward to see that expanding and contracting backgrounds are identified by $k>0$ and $k<0$ respectively. $k=0$ corresponds to the flat $(d+1)$-dimensional Minkowski space. Since we are interested in expanding backgrounds here and throughout the paper we shall consider only positive values of $k$, i.e. solutions with $\Pi_{\Theta}>0$. For $q \leqslant 1$ we have two distinct branches corresponding to PRBB and POBB states. (The limiting case $q=1$ corresponds to a positive constant potential in (2.1). The relative classical solutions have been discussed in [17].) The PRBB (+) and POBB (-) branches are identified by negative and positive values of $\Pi_{\Phi}$, respectively. Asymptotically, for $0<q \leqslant 1$ we have

$$
\lim _{\Phi \rightarrow+\infty} \Pi_{\Phi}^{( \pm)}=\mp k, \quad \lim _{\Phi \rightarrow-\infty} \Pi_{\Phi}^{( \pm)} \sim \mp \lambda_{s} \sqrt{\lambda} \mathrm{e}^{-q \Phi},
$$

in the strong- and weak-coupling regime, respectively. Conversely, for $q<0$ we have

$$
\lim _{\Phi \rightarrow+\infty} \Pi_{\Phi}^{( \pm)} \sim \mp \lambda_{s} \sqrt{\lambda} \mathrm{e}^{-q \Phi}, \quad \lim _{\Phi \rightarrow-\infty} \Pi_{\Phi}^{( \pm)}=\mp k .
$$

Substituting (2.15) in (2.13) the synchronous-gauge time $t^{(s g)}$ can be written explicitly in terms of $\tau$. There are two different cases:

(a) $q \neq 1 /(2 m+1), m=0,1,2, \ldots$

$$
\begin{aligned}
t^{(s g)}-t_{0}^{(s g)}= & -\frac{\sigma(q) \sigma\left(\tau-\tau_{0}\right)}{\sqrt{\lambda}|q-1|}\left(\frac{\sqrt{\lambda} \lambda_{s}}{k}\right)^{1-1 / q} \\
& \times[\sinh (x)]^{1-1 / q} F\left(\frac{1}{2}, \frac{q-1}{2 q}, \frac{3 q-1}{2 q},-\sinh ^{2} x\right),
\end{aligned}
$$

where $\sigma$ is the sign function, $F$ is the hypergeometric function [20], and

$$
x=\frac{k}{\lambda_{s}}\left|q\left(\tau-\tau_{0}\right)\right|
$$

(b) $q=1 /(2 m+1), m=0,1,2, \ldots$

$$
t^{(s g)}-t_{0}^{(s g)}=-\frac{\sigma\left(\tau-\tau_{0}\right)}{2 \sqrt{\lambda}|q|}\left(\frac{\sqrt{\lambda} \lambda_{s}}{k}\right)^{1-1 / q} \frac{\Gamma(m+1 / 2)}{\sqrt{\pi} \Gamma(m+1)} f(m, x),
$$

where

$$
f(0, x)=4 \operatorname{arcoth}\left(\mathrm{e}^{x}\right),
$$

and

$$
f(m, x)=(-1)^{m} f(0, x)+\cosh (x) \sum_{k=0}^{m-1} \frac{(-1)^{k} \Gamma(m-k) \sqrt{\pi}}{\Gamma\left(m+\frac{1}{2}-k\right)[\sinh (x)]^{2(m-k)}}
$$

for $m>0$.

The above relations determine the PRBB and POBB branches in terms of the synchronousgauge time $t^{(s g)}$ for different values of the parameter $q$. In particular, we have: 
(a) $q<0$. In this case the PRBB and POBB branches are defined for

$$
\begin{aligned}
& -\infty<\tau-\tau_{0}<0 \quad \rightarrow \quad-\infty<t^{(s g)}-t_{0}^{(s g)}<0, \\
& 0<\tau-\tau_{0}<\infty \quad \rightarrow \quad 0<t^{(s g)}-t_{0}^{(s g)}<\infty
\end{aligned}
$$

respectively.

(b) $q=1 /(2 m+1)$. The PRBB and the POBB branches are defined for

$$
\begin{aligned}
& 0<\tau-\tau_{0}<\infty \quad \rightarrow \quad-\infty<t^{(s g)}-t_{0}^{(s g)}<0, \\
& -\infty<\tau-\tau_{0}<0 \rightarrow 0<t^{(s g)}-t_{0}^{(s g)}<\infty,
\end{aligned}
$$

respectively. This can be checked using the asymptotic expansions of $f(m, x)$ for $x \rightarrow 0$ and $x \rightarrow+\infty$.

(c) $0<q<1, q \neq 1 /(2 m+1)$. The PRBB branch is defined for

$$
0<\tau-\tau_{0}<\infty \rightarrow \infty<t^{(s g)}-t_{0}^{(s g)}<-T,
$$

and the POBB branch for

$$
-\infty<\tau-\tau_{0}<0 \rightarrow T<t^{(s g)}-t_{0}^{(s g)}<\infty
$$

where

$$
T=\frac{1}{2 \sqrt{\pi}|q|}\left(\frac{\sqrt{\lambda} \lambda_{s}}{k}\right)^{1-1 / q} \Gamma\left(\frac{q-1}{2 q}\right) \Gamma\left(\frac{1}{2 q}\right) .
$$

As has been pointed out in [8,9], in terms of the synchronous-gauge time $t^{(s g)}$ the $\mathrm{PRBB}$ and POBB branches are separated by a finite interval $\Delta t^{(s g)}=2 T$. However, the separation between the two branches has no physical meaning. Indeed, due to the presence of a singularity in the curvature and in the string coupling the PRBB and POBB solutions are disjoint. Therefore, it is possible to define the initial value of $t$ such that the singularity occurs at $t^{(\mathrm{sg})}=0$ in both branches.

\section{Quantum theory}

The string cosmological model of section 2 is described by a time-reparametrization-invariant Hamiltonian system with two degrees of freedom. Though its quantization involves subtleties typical of gauge-invariant systems $[10,11,18]$, the standard techniques of quantization of constrained systems can be applied straightforwardly thanks to the integrability properties of the model [12-14].

The starting point is the canonical action (2.8). Since the constraint $H$ is of the form $H=H_{\Theta}(\Theta)+H_{\Phi}(\Phi)$, the time parameter can be defined by a single degree of freedom. In the previous section we have seen that the sign of $\Pi_{\Theta}$ determines the contracting versus expanding behaviour of the solutions and the sign of $\Pi_{\Phi}$ identifies the PRBB versus POBB phases. Since we are interested in the calculation of the quantum transition probability from an (expanding) PRBB phase to an (expanding) POBB phase, it is natural to use the $\left(\Theta, \Pi_{\Theta}\right)$ degree of freedom to define the time of the system and fix the gauge. In this case, the eigenstates of the effective Hamiltonian are identified by a continuous quantum number $k$ corresponding to the classical value of $\Pi_{\Theta}$. Wavefunctions that describe expanding (contracting) solutions are eigenstates of the effective Hamiltonian with $k>0(k<0)$.

Let us consider the canonical transformation $[10]\left(\Theta, \Pi_{\Theta}, \Phi, \Pi_{\Phi}\right) \rightarrow\left(\Sigma, \Pi_{\Sigma}, \Phi, \Pi_{\Phi}\right)$ where

$$
\Sigma=\lambda_{s} \frac{\Theta}{\Pi_{\Theta}}, \quad \Pi_{\Sigma}=\frac{1}{2 \lambda_{s}} \Pi_{\Theta}^{2} .
$$


In terms of the new canonical variables the constraint (2.9) reads

$$
H\left(\Sigma, \Pi_{\Sigma}, \Phi, \Pi_{\Phi}\right)=\Pi_{\Sigma}-\frac{1}{2 \lambda_{s}}\left[\Pi_{\Phi}^{2}-\lambda \lambda_{s}^{2} \mathrm{e}^{-2 q \Phi}\right]=0 .
$$

From (3.2) it is straightforward to see that $\Sigma$ is canonically conjugate to $H$. Thus it defines a global time parameter $[11,19]$. In particular, the gauge-fixing identity can be chosen as

$$
F(\Sigma ; t) \equiv \Sigma+t=0 \text {. }
$$

Equation (3.3) fixes the Lagrange multiplier as $\mu=-1$. The gauge-fixed action reads

$$
S_{\mathrm{eff}}=\int_{t_{1}}^{t_{2}} \mathrm{~d} t\left[\dot{\Phi} \Pi_{\Phi}-H_{\mathrm{eff}}\left(\Phi, \Pi_{\Phi}\right)\right],
$$

where the effective Hamiltonian is

$$
H_{\text {eff }}\left(\Phi, \Pi_{\Phi}\right)=\frac{1}{2 \lambda_{s}}\left(\Pi_{\Phi}^{2}-\lambda \lambda_{s}^{2} \mathrm{e}^{-2 q \Phi}\right) .
$$

The system described by the effective Hamiltonian (3.5) is free of gauge degrees of freedom and its quantization can be performed using the standard techniques. In the next two subsections we shall discuss the reduced phase space and path-integral quantization procedures.

\subsection{Reduced phase space quantization}

The reduced phase space is described by a single degree of freedom with canonical coordinates $\left(\Phi \in \mathbb{R}, \Pi_{\Phi} \in \mathbb{R}\right)$. Thus there are no ambiguities in the choice of the measure in the Hilbert space: $\mathrm{d}[\mu]=\mathrm{d} \Phi$. In the standard operator approach the quantization of the model is obtained by identifying the canonical coordinates with operators. In the Schrödinger representation the self-adjoint operators with respect to the measure $\mathrm{d}[\mu]$ are

$$
\Phi \rightarrow \hat{\Phi}=\Phi, \quad \Pi_{\Phi} \rightarrow \hat{\Pi}_{\Phi}=-\mathrm{i} \frac{\partial}{\partial \Phi} .
$$

Since the effective Hamiltonian is quadratic in the momenta there are no factor ordering ambiguities. The Schrödinger equation reads

$$
-\mathrm{i} \frac{\partial}{\partial t} \Psi(\Phi ; t)=\frac{1}{2 \lambda_{s}}\left[\frac{\partial^{2}}{\partial \Phi^{2}}+\lambda \lambda_{s}^{2} \mathrm{e}^{-2 q \Phi}\right] \Psi(\Phi ; t),
$$

where $t$ is defined by (3.3). Finally, the inner product in the Hilbert space is

$$
\left(\Psi_{2}, \Psi_{1}\right)=\int_{-\infty}^{+\infty} \mathrm{d} \Phi \Psi_{2}^{*}(\Phi ; t) \Psi_{1}(\Phi ; t) .
$$

The general solution of the Schrödinger equation (3.7) can be written as

$$
\Psi_{q}(\Phi ; t)=\int \mathrm{d} k A(k) \psi_{k, q}(\Phi) \mathrm{e}^{-\mathrm{i} k^{2} t / 2 \lambda_{s}},
$$

where $\psi_{k, q}(\Phi)$ is the solution of the stationary Schrödinger equation $\hat{H}_{\text {eff }} \psi=E \psi$ with energy $E=k^{2} / 2 \lambda_{s}$

$$
\left[\frac{\mathrm{d}^{2}}{\mathrm{~d}^{2}}+\lambda \lambda_{s}^{2} \mathrm{e}^{-2 q \Phi}\right] \psi_{k, q}(\Phi)=-k^{2} \psi_{k, q}(\Phi) .
$$

For $q \neq 0$ we have

$$
\psi_{k, q}(z)=A_{1}(k, q) J_{i v}(z)+A_{2}(k, q) Y_{i v}(z),
$$


where $A_{1}(k, q)$ and $A_{2}(k, q)$ are arbitrary functions, and $J_{i v}(z), Y_{i v}(z)$ are the Bessel functions of the first and second kind of index $i v=i|k / q|$ and argument

$$
z=\sqrt{\lambda} \lambda_{s} \exp (-q \Phi) /|q|,
$$

respectively [20].

Since the space of the solutions (3.11) is two dimensional we have two sets of (real) orthonormal functions with respect to the inner product $(3.8)[10,21]$

$$
\begin{aligned}
& \chi_{v}^{(1)}(z)=C^{(1)}\left[\mathrm{e}^{-\pi v / 2} H_{i v}^{(1)}(z)+\mathrm{e}^{\pi v / 2} H_{i v}^{(2)}(z)\right], \\
& \chi_{v}^{(2)}(z)=\mathrm{i} C^{(2)}\left[\mathrm{e}^{-\pi v / 2} H_{i v}^{(1)}(z)-\mathrm{e}^{\pi v / 2} H_{i v}^{(2)}(z)\right],
\end{aligned}
$$

where

$$
C^{(1)}=\sqrt{\frac{\nu \cosh (\pi v / 2)}{4 \sinh (\pi \nu / 2)}}, \quad C^{(2)}=\sqrt{\frac{\nu \sinh (\pi \nu / 2)}{4 \cosh (\pi \nu / 2)}},
$$

and $H_{i v}^{(1)}(z)$ and $H_{i v}^{(2)}(z)$ are the Hankel functions of the first and second kind, respectively, [20].

Now let us identify the stationary wavefunctions that correspond to expanding PRBB and POBB phases. We discuss in detail the case $0<q \leqslant 1$ leaving to the end of this subsection the discussion of negative values of $q$.

From (3.1) and (3.2) it follows that $\frac{1}{2 \lambda_{s}} \Pi_{\Theta}^{2}=H_{\text {eff }}$. Thus phases that are expanding (contracting) are described by eigenstates of the effective Hamiltonian with $k>0(k<$ 0 ). Expanding wavefunctions that correspond to PRBB and POBB can be identified by investigating the asymptotic behaviours of (3.13) and (3.14) in the weak- and strong-coupling regimes. For $z \rightarrow \infty$, i.e. in the weak-coupling regime, the wavefunctions (3.13) and (3.14) behave as

$$
\begin{aligned}
& \chi_{v}^{(1)}(z) \approx \sqrt{\frac{2}{\pi z}} C^{(1)}\left[\mathrm{e}^{\mathrm{i}(z-\pi / 4)}+\mathrm{e}^{-\mathrm{i}(z-\pi / 4)}\right], \\
& \chi_{v}^{(2)}(z) \approx \mathrm{i} \sqrt{\frac{2}{\pi z}} C^{(2)}\left[\mathrm{e}^{\mathrm{i}(z-\pi / 4)}-\mathrm{e}^{-\mathrm{i}(z-\pi / 4)}\right] .
\end{aligned}
$$

By applying the momentum operator $\hat{\Pi}_{\Phi}$ to the linear combinations $\chi_{v}^{( \pm)}=C^{(2)} \chi_{v}^{(1)} \mp \mathrm{i} C^{(1)} \chi_{v}^{(2)}$ we find

$$
\hat{\Pi}_{\Phi} \chi_{\nu}^{( \pm)} \sim \mp \lambda_{s} \sqrt{\lambda} \mathrm{e}^{-q \Phi} \chi_{\nu}^{( \pm)} .
$$

Thus the wavefunctions corresponding to PRBB and POBB in the weak-coupling regime are proportional to the linear combinations $\chi_{v}^{( \pm)}$, respectively. The normalized PRBB and POBB wavefunctions in the weak-coupling regime are

$$
\psi_{W}^{( \pm)}=\frac{1}{\sqrt{2 \operatorname{coth}(\pi v)}}\left[\sqrt{\tanh (\pi v / 2)} \chi_{v}^{(1)} \mp \mathrm{i} \sqrt{\operatorname{coth}(\pi \nu / 2)} \chi_{\nu}^{(2)}\right] .
$$

By a similar argument we find that the normalized wavefunctions that correspond to expanding $\mathrm{PRBB}$ and $\mathrm{POBB}$ phases in the strong-coupling regime are

$$
\psi_{S}^{( \pm)}=\frac{1}{\sqrt{2}}\left[\chi_{v}^{(1)} \mp \mathrm{i} \chi_{v}^{(2)}\right] .
$$

Using the two sets of wavefunctions (3.19) and (3.20) it is possible to compute the amplitudes that correspond to the different transitions. They are schematically represented in figure 1, 


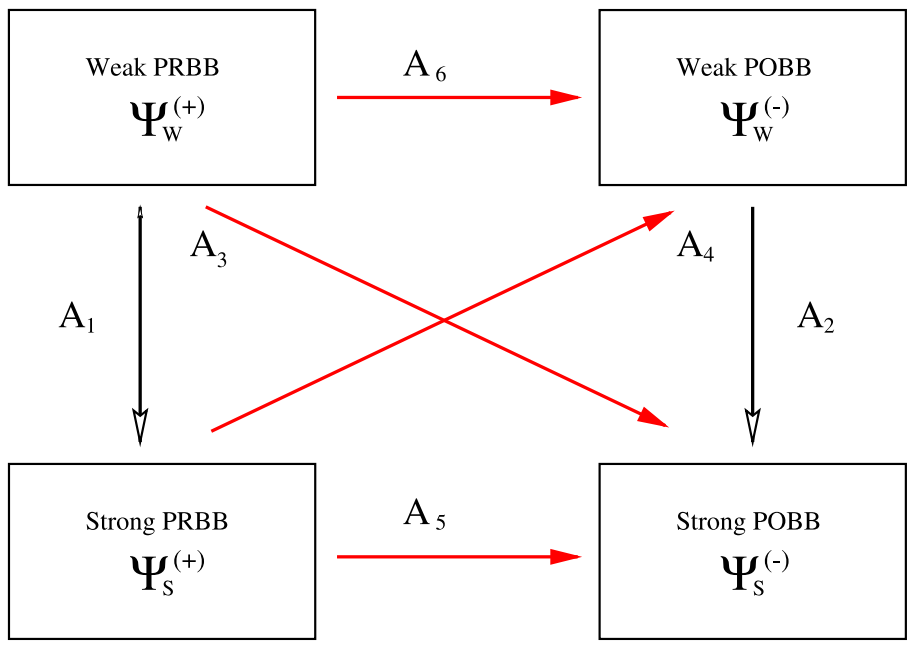

$\longrightarrow$ Classically allowed transition

Classically forbidden transition

Figure 1. Different transitions in the weak- and strong-coupling regimes.

where the amplitudes $A_{1}, \ldots, A_{6}$ are given by the following expressions:

$$
\begin{aligned}
& A_{1} \equiv\left(\psi_{S}^{(+)}, \psi_{W}^{(+)}\right)=\frac{1}{\sqrt{1+\mathrm{e}^{-2 \pi k / q}}}, \\
& A_{2} \equiv\left(\psi_{S}^{(-)}, \psi_{W}^{(-)}\right)=\frac{1}{\sqrt{1+\mathrm{e}^{-2 \pi k / q}}}, \\
& A_{3} \equiv\left(\psi_{S}^{(-)}, \psi_{W}^{(+)}\right)=\frac{-\mathrm{e}^{-\pi k / q}}{\sqrt{1+\mathrm{e}^{-2 \pi k / q}}}, \\
& A_{4} \equiv\left(\psi_{W}^{(-)}, \psi_{S}^{(+)}\right)=\frac{-\mathrm{e}^{-\pi k / q}}{\sqrt{1+\mathrm{e}^{-2 \pi k / q}}}, \\
& A_{5} \equiv\left(\psi_{S}^{(-)}, \psi_{S}^{(+)}\right)=0, \\
& A_{6} \equiv\left(\psi_{W}^{(-)}, \psi_{W}^{(+)}\right)=\frac{-2 \mathrm{e}^{-\pi k / q}}{1+\mathrm{e}^{-2 \pi k / q}} .
\end{aligned}
$$

Let us discuss in depth the transition amplitudes (3.21). The amplitudes $A_{1}$ and $A_{2}$ correspond to classically allowed transitions. The relative transition probabilities $\left(P_{S, W}^{(+,+)} \equiv\left|A_{1}\right|^{2}\right.$, $\left.P_{S, W}^{(-,-)} \equiv\left|A_{2}\right|^{2}\right)$ are

$$
P_{S, W}^{(+,+)}=P_{S, W}^{(-,-)}=\frac{1}{1+\mathrm{e}^{-2 \pi k / q}} .
$$

For $k \rightarrow \infty$, i.e. in the semiclassical limit, (3.22) becomes

$$
P_{S, W}^{(+,+)}=P_{S, W}^{(-,-)} \sim 1+\mathrm{O}\left(\mathrm{e}^{-2 \pi k / q}\right),
$$


in agreement with the classical theory. The amplitudes $A_{3}$ and $A_{4}$ describe classically forbidden transitions. The relative transition probabilities $\left(P_{W, S}^{(+,-)} \equiv\left|A_{3}\right|^{2}, P_{S, W}^{(+,-)} \equiv\left|A_{4}\right|^{2}\right)$ are

$$
P_{W, S}^{(+,-)}=P_{S, W}^{(+,-)}=\frac{\mathrm{e}^{-2 \pi k / q}}{1+\mathrm{e}^{-2 \pi k / q}} .
$$

In the semiclassical limit (3.24) becomes

$$
P_{W, S}^{(+,-)}=P_{S, W}^{(+,-)} \sim \mathrm{e}^{-2 \pi k / q}+\mathrm{O}\left(\mathrm{e}^{-4 \pi k / q}\right) .
$$

The transition probabilities (3.24) become highly suppressed for $k \gg 1$ where the evolution follows essentially the classical trajectory. In the limit $k \rightarrow 0$ equations (3.22) and (3.24) become

$$
P_{S, W}^{(+,+)}=P_{S, W}^{(-,-)} \sim P_{W, S}^{(+,-)}=P_{S, W}^{(+,-)} \sim \frac{1}{2}+\mathrm{O}(k) .
$$

In the small- $k$ limit quantum effects are significant: the PRBB (POBB) phase in the weakcoupling regime has the same probability of evolving in the PRBB or in the POBB phase in the strong-coupling regime (and vice versa).

The probability of the transition $\mathrm{PRBB} \rightarrow \mathrm{POBB}$ in the strong-coupling regime $\left(P_{S, S}^{(+,-)}=\right.$ $\left.\left|A_{5}\right|^{2}\right)$ is identically zero. This can be understood looking at the asymptotic form of the potential for $\Phi \rightarrow \infty(z \rightarrow 0)$. Indeed, for large values of $\Phi$ the potential term in (3.10) goes asymptotically to zero. Consequently, PRBB and POBB wavefunctions in the strongcoupling regime behave asymptotically as free plane waves with opposite $\Pi_{\Phi}$ momentum. Since reflection of free plane waves is forbidden, the quantum transition from PRBB to POBB in the strong-coupling regime does not take place.

The last and most interesting result is the probability of transition from the PRBB phase in the weak-coupling regime to the POBB phase in the weak-coupling regime

$$
P_{W, W}^{(+,-)} \equiv\left|A_{6}\right|^{2}=4 \frac{\mathrm{e}^{-2 \pi k / q}}{\left(1+\mathrm{e}^{-2 \pi k / q}\right)^{2}} .
$$

The semiclassical limit of (3.27) is

$$
P_{W, W}^{(+,-)} \sim 4 \mathrm{e}^{-2 \pi k / q}+\mathrm{O}\left(\mathrm{e}^{-4 \pi k / q}\right) .
$$

For $q=1$ the semiclassical result coincides, apart from a normalization factor, with the 'reflection coefficient' of $[7,8]$. However, the result of $[7,8]$ should be considered as a ratio between two different transition probabilities rather than a transition probability by itself. Precisely, the reflection coefficient defined in $[7,8]$ is

$$
R \equiv \frac{P_{S, W}^{(-,+)}}{P_{S, W}^{(+,+)}}=\mathrm{e}^{-2 \pi k / q} .
$$

Note that the (classically forbidden) transition from the strong-coupling PRBB phase to the weak-coupling POBB phase is suppressed by a factor of $\mathrm{e}^{-2 \pi k / q}$ with respect to the (classically allowed) transition from the strong-coupling PRBB phase to the weak-coupling PRBB phase.

Equations (3.23), (3.25) and (3.28) also give the asymptotic behaviours for small values of $q$ at given $k$. In this case quantum effects are negligible. When $0<q \ll 1$, the potential in the Schrödinger equation is nearly constant and the PRBB and POBB solutions are approximated by plane waves of opposite momentum along $\Phi$. In this case reflection of waves is highly suppressed.

A similar analysis can be performed for negative values of $q$. For $q<0$ the wavefunctions that correspond to expanding PRBB and POBB phases are

$$
\begin{aligned}
& \psi_{W}^{( \pm)}=\frac{1}{\sqrt{2}}\left[\chi_{\nu}^{(1)} \pm \mathrm{i} \chi_{v}^{(2)}\right], \\
& \psi_{S}^{( \pm)}=\frac{1}{\sqrt{2 \operatorname{coth}(\pi v)}}\left[\sqrt{\tanh (\pi \nu / 2)} \chi_{v}^{(1)} \pm \mathrm{i} \sqrt{\operatorname{coth}(\pi v / 2)} \chi_{v}^{(2)}\right] .
\end{aligned}
$$


The amplitudes for the various transitions can be read from (3.21) with the substitutions $A_{1} \leftrightarrow A_{2}, A_{5} \leftrightarrow A_{6}$ and $q \rightarrow-q$. Now the transition from the weak-coupling PRBB phase to the strong-coupling POBB phase is forbidden for negative values of $q$.

The results from this section show that the probabilities of classically forbidden transitions can be expressed, in the semiclassical limit, as power series of $\mathrm{e}^{-2 \pi k /|q|}$. Following $[7,8]$, from (2.18) and (2.4) we find

$$
\exp (-2 \pi k /|q|)=\exp \left(\frac{-\sqrt{4 d} \pi \Omega_{s}}{|q| g_{s}^{2} \lambda_{s}^{d}}\right),
$$

where $\Omega_{s}$ is the proper spatial volume and $g_{s}=\mathrm{e}^{\phi_{s} / 2}$ is the value of the string coupling when $\boldsymbol{H}=1 / \lambda_{s}$. The 'instanton-like' behaviour of (3.30) shows that the probabilities of classically forbidden transitions are peaked in the strong-coupling regime-as has already been pointed out in $[7,8]$ - where all powers of $\mathrm{e}^{-2 \pi k /|q|}$ have to be taken into account. The occurrence of this instanton-like behaviour will be clarified in the next subsection.

\subsection{Path-integral quantization}

The string cosmology model that we are considering can also be quantized using the functional approach. The aim of this subsection is to show how to compute, using the path-integral formalism, the probability $P_{W, W}^{(+,-)}$in the semiclassical limit. While in the case under investigation the semiclassical path-integral calculation seems devoid of interest-we already know the exact transition probability (3.27) -nevertheless, the semiclassical calculation is of primary importance if the system cannot be quantized exactly. We shall show that the functional approach, when performed appropriately, reproduces the exact result in the limit of large $k$. So it seems not unreasonable to assume that the semiclassical path-integral calculation also gives a sound approximation of the exact result for those models that are not exactly solvable. In future, we aim to apply the formalism of this subsection to more realistic and interesting models of string cosmology.

The starting point of the functional approach is the path-integral in the reduced space $[14,22]$

$$
I=\int_{\Phi\left(t_{1}\right)}^{\Phi\left(t_{2}\right)} \mathcal{D} \Phi \mathcal{D} \Pi_{\Phi} \exp \left(\mathrm{i} S_{\mathrm{eff}}\left[\Phi, \Pi_{\Phi}\right]\right)
$$

where the effective action is given by (3.4) and (3.5). The transition amplitude $A_{6}$ is defined by (3.31) where the integral is evaluated on all paths that satisfy the boundary conditions

$$
\Phi(-\infty)=-\infty, \quad \Phi(\infty)=-\infty .
$$

Since the effective Hamiltonian is quadratic in $\Pi_{\Phi}$ the integral in $\Pi_{\Phi}$ can be evaluated immediately. We obtain

$$
I=\int_{\Phi\left(t_{1}\right)}^{\Phi\left(t_{2}\right)} \mathcal{D} \Phi \exp \left(\mathrm{i} \int_{t_{1}}^{t_{2}} \mathrm{~d} t \mathcal{L}_{\mathrm{eff}}[\Phi, \dot{\Phi}]\right)
$$

where the effective Lagrangian is

$$
\mathcal{L}_{\text {eff }}=\frac{1}{2} \lambda_{s}\left(\dot{\Phi}^{2}+\lambda \mathrm{e}^{-2 q \Phi}\right) .
$$

It is advisable to use the variable $z$ defined in (3.12). Equation (3.34) becomes

$$
\mathcal{L}_{\text {eff }}=\frac{1}{2 \lambda_{s}}\left(\frac{\lambda_{s}^{2}}{q^{2}} \frac{\dot{z}^{2}}{z^{2}}+q^{2} z^{2}\right)
$$


Let us first consider the case where $0<q \leqslant 1$. The path integral (3.33) must be evaluated on all trajectories that satisfy the boundary conditions

$$
z(-\infty)=\infty, \quad z(\infty)=\infty .
$$

The effective Lagrangian (3.35) is singular when $z=0$. So there are no classical solutions describing a (smooth) transition between PRBB and POBB phases (see section 2). However, it is possible to construct quasi-classical trajectories that satisfy the boundary conditions (3.36) and interpolate between the PRBB and POBB phases.

Let us consider the analytical continuation of the variable $z$ into the complex plane. The effective Lagrangian is analytical in any point of the complex plane $(\operatorname{Re}(z), \operatorname{Im}(z))$ except for $z=0$. Classically, the transition from the weak-coupling PRBB phase to the weak-coupling POBB phase would correspond to the trajectory starting at $z=+\infty$, going left along the real axis (PRBB phase, $\dot{\Phi}>0$ ), reaching the origin, and finally going right along the real axis to $z=+\infty$ (POBB phase, $\dot{\Phi}<0$ ). Clearly, since the Lagrangian is singular in $z=0$ a classical continuous and differentiable solution does not exist.

Now consider generic analytical trajectories in the complex plane that start at $\operatorname{Re}(z)=\infty$, $\operatorname{Im}(z)>0$, and end at $\operatorname{Re}(z)=\infty, \operatorname{Im}(z)<0$ (see figure $2(a)$ ). We can divide this class of trajectories into three (topologically) distinct categories.

- Trajectories that do not cross the imaginary axis, i.e. trajectories that cross the real axis at (at least) one point $z=z_{0}, \operatorname{Re}\left(z_{0}\right)>0, \operatorname{Im}\left(z_{0}\right)=0$ (curve $\gamma_{0}$ in figure $2(a)$ ).
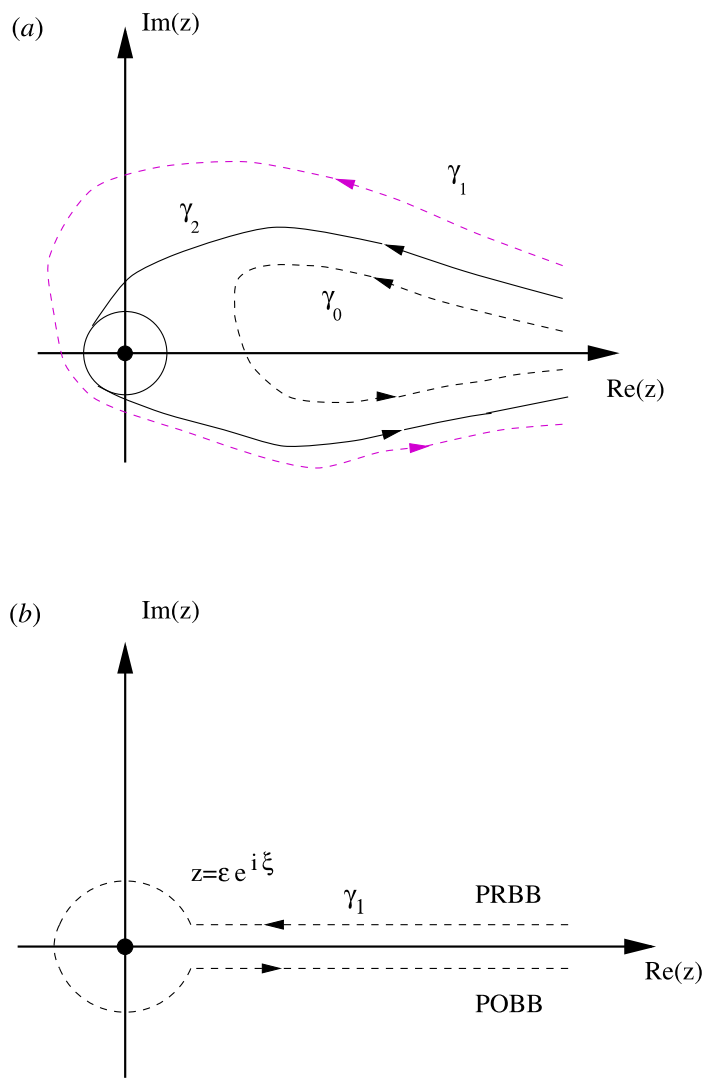

Figure 2. Contours of integration in the complex $z$-plane. 
- Trajectories that cross the imaginary axis twice, i.e. trajectories that cross the real axis once in $z=z_{0}, \operatorname{Re}\left(z_{0}\right)<0, \operatorname{Im}\left(z_{0}\right)=0$ (curve $\gamma_{1}$ in figure $2(a)$ ).

- Trajectories that cross the imaginary axis $2 n$ times $(n=2,3, \ldots)$, i.e. trajectories that cross the positive real axis $n-1$ times and the negative real axis $n$ times (curve $\gamma_{n}$ in figure 2(a) for $n=2$ ).

Since the action is analytical over the entire complex plane except for $z=0$ trajectories of type $\gamma_{0}$ can be deformed continuously to a (two-folded) trajectory lying entirely on the real positive axis and defined in the interval $\left(\operatorname{Re}\left(z_{0}\right), \infty\right)$. These curves correspond to classical solutions with the dilaton field evolving from $\Phi=-\infty$ to a maximum value $\Phi=-\ln \left[q \operatorname{Re}\left(z_{0}\right) / \sqrt{\lambda} \lambda_{s}\right] / q$ and then decreasing to $\Phi=-\infty$. A straightforward calculation shows that the action evaluated on this path is identically zero. Since (3.34) is positive definite $S_{\text {eff }}=0$ can be obtained only by a time reflection, i.e. by a PRBB (POBB) phase that is covered twice. Therefore, these trajectories do not describe transitions from PRBB to POBB phases.

Let us focus our attention on trajectories of type $\gamma_{1}$. They can be deformed continuously to a trajectory that lies entirely on the real positive axis except around $z=0$, where the singularity is avoided by the (small) circle $z=\varepsilon \mathrm{e}^{\mathrm{i} \xi}, \varepsilon \rightarrow 0,0 \leqslant \xi<2 \pi$ (see figure 2(b)). This trajectory describes a transition from the weak-coupling PRBB phase to the weak-coupling POBB phase and corresponds to a classical solution except in a small region in the strong-coupling limit, where the singularity of the classical solution is avoided by the analytical continuation in the complex plane. We shall see that the path-integral evaluated on this trajectory gives the leading contribution to the semiclassical approximation of the transition amplitude $A_{6}$. Trajectories of type $\gamma_{n}$ (with $n>1$ ) give contributions of higher order.

It is worth spending a few words on examining the meaning of the analytical continuation of the variable $z$ in the complex plane. Setting $z=R \mathrm{e}^{\mathrm{i} \xi}$ and using (2.2) and (3.12) the metric is cast in the form

$$
\mathrm{d} s^{2}=-\left(\frac{q R}{\sqrt{\lambda} \lambda_{s}}\right)^{2 / q} \mathrm{e}^{2 \mathrm{i} \xi / q} \mu^{2} \mathrm{~d} t^{2}+a^{2}(t) \mathrm{d} x_{i} \mathrm{~d} x^{i} .
$$

The signature of (3.37) is a function of $\xi$. In particular, the metric (3.37) is real hyperbolic for $\xi=\pi q n$ and real Riemannian for $\xi=\pi q(2 n+1) / 2$, where $n$ is an integer number. Therefore, the analytic continuation of figure 2(b) can be interpreted as a sort of Euclidean analytical continuation in the space of metrics. Any trajectory that circles $z=0$ can be considered as an ' $n$-instanton' solution (with no well defined signature) labelled by a winding number $n$ that corresponds to the number of times that the trajectory wraps around the singularity in $z=0$. In the semiclassical limit, the transition amplitude $P_{W, W}^{(+,-)}$is given by the path-integral (3.33) evaluated on the class of $n$-instanton solutions.

Let us consider the contribution to (3.33) of the one-instanton solution

$$
I_{\mathrm{sc}}^{(1)}=C_{1} \exp \left(\mathrm{i} S_{\mathrm{eff}}\left[z_{\gamma_{1}}, \dot{z}_{\gamma_{1}}\right]\right),
$$

where $C_{1}$ is a normalization factor and the subscript $\gamma_{1}$ means that the effective action is evaluated along the curve $\gamma_{1}$ of figure $2(b)$. For a trajectory with energy $k^{2} / 2 \lambda_{s}$ the effective action can be cast into the form

$$
S_{\text {eff }}=\int_{\gamma_{1}} \frac{\mathrm{d} z}{z} \frac{1}{\sqrt{z^{2}+k^{2} / q^{2}}}\left(z^{2}+k^{2} / 2 q^{2}\right) .
$$

As we expect, the effective Lagrangian has one isolated singularity at $z=0$ (pole of order one). Moreover, for $z \rightarrow \infty$ the action (3.39) shows a linear divergence. The latter is due to the asymptotic behaviour of the PRBB and POBB wavefunctions in the weak-coupling 
regime. Indeed, using (3.38) and (3.39) the wavefunctions corresponding to the PRBB and POBB phases in the semiclassical approximation are

$$
\begin{aligned}
& \psi^{(+)} \sim \exp \left[\mathrm{i} \int_{\infty}^{z} \frac{\mathrm{d} z^{\prime}}{z^{\prime}} \frac{1}{\sqrt{z^{\prime 2}+k^{2} / q^{2}}}\left(z^{\prime 2}+k^{2} / 2 q^{2}\right)\right], \\
& \psi^{(-)} \sim \exp \left[\mathrm{i} \int_{z}^{\infty} \frac{\mathrm{d} z^{\prime}}{z^{\prime}} \frac{1}{\sqrt{z^{\prime 2}+k^{2} / q^{2}}}\left(z^{\prime 2}+k^{2} / 2 q^{2}\right)\right] .
\end{aligned}
$$

In the weak-coupling regime $(\operatorname{Im}(z)=0, z \rightarrow \infty)$, equations (3.40) and (3.41) behave asymptotically as

$$
\psi_{z \rightarrow \infty}^{(+)} \sim \mathrm{e}^{\mathrm{i} z}, \quad \psi_{z \rightarrow \infty}^{(-)} \sim \mathrm{e}^{-\mathrm{i} z},
$$

in agreement with the asymptotic behaviour of (3.20).

The integral (3.39) can be made convergent by subtracting the asymptotic phase contribution for $z \rightarrow \infty$. Then, using the residue theorem, we obtain $S_{\text {eff }}=\pi \mathrm{i} k / q$. The amplitude (3.38) is given by

$$
I_{\mathrm{sc}}^{(1)}=C_{1} \mathrm{e}^{-\pi k / q} .
$$

The semiclassical one-instanton amplitude (3.43) approximates the (exact) result for large values of $k$. This proves the consistency of the reduced phase space and path-integral quantization methods. The contribution of the $n$-instanton $(n>1)$ to the transition amplitude $A_{6}$ is

$$
I_{\mathrm{sc}}^{(n)}=C_{n} \mathrm{e}^{-\pi n k / q}
$$

Hence, $n$-instanton terms give higher-order contributions in the large- $k$ expansion. Equations (3.43) and (3.44) show that the instanton-like dependence (3.30) on the string coupling constant of the amplitudes that correspond to classically forbidden transitions can be traced back to the existence, in the semiclassical regime, of trajectories that connect smoothly the PRBB and POBB phases.

Let us conclude this section with two remarks. In the computation of (3.39) we have chosen only anticlockwise trajectories (see figures $1(a)$ and $(b)$ ). If we considered clockwise paths the residue theorem would give $S_{\text {eff }}=-\pi \mathrm{i} k / q$ and the generic contribution to the transition amplitude would be

$$
\tilde{I}_{\mathrm{sc}}^{(n)}=C_{n} \mathrm{e}^{\pi n k / q} .
$$

This result violates, in the semiclassical limit, the unitarity bound. However, there is a simple argument that allows one to remove this pathology. Let us consider the asymptotic behaviour of PRBB and POBB wavefunctions in the weak-coupling regime. For complex values of $z$ (3.42) read

$$
\psi^{(+)}(z \rightarrow \infty) \sim \mathrm{e}^{\mathrm{i} \operatorname{Re}(z)-\operatorname{Im}(z)}, \quad \psi^{(-)}(z \rightarrow \infty) \sim \mathrm{e}^{-\mathrm{i} \operatorname{Re}(z)+\operatorname{Im}(z)} .
$$

Since the system must be classical in the weak-coupling regime, the contribution to the pathintegral of the trajectories that approach the real axis for $z \rightarrow \infty$ must dominate the contribution of the trajectories with a non-zero value of $\operatorname{Im}(z)$. The above requirement is verified if we integrate along the anticlockwise trajectories. (In this case the PRBB and POBB branches are identified by $\operatorname{Im}(z)>0$ and $\operatorname{Im}(z)<0$, respectively.)

For $q<0$ the transition amplitude $A_{6}$ is identically zero. Indeed, setting $w=1 / z$, the effective Lagrangian (3.35) becomes

$$
\mathcal{L}_{\text {eff }}=\frac{1}{2 \lambda_{s}}\left(\frac{\lambda_{s}^{2}}{q^{2}} \frac{\dot{w}^{2}}{w^{2}}+\frac{q^{2}}{w^{2}}\right)
$$


and the path integral (3.33) must be evaluated on trajectories that satisfy the boundary conditions $w(-\infty)=\infty, w(\infty)=\infty$. The action evaluated on a generic $n$-instanton solution is identically zero. Therefore, the semiclassical trajectories do not correspond to a transition between PRBB and POBB phases.

\section{Conclusions}

The graceful exit, i.e. the transition from the inflationary 'pre-big-bang phase' to the deflationary 'post-big-bang' phase is a fundamental subject of research in (quantum) string cosmology.

In this paper we have addressed this topic by investigating a special class of minisuperspace models that are invariant under scale factor duality transformations. Though this particular class of models had been considered previously in the literature [7-9] a deeper discussion was needed. Indeed, our analysis clarifies some issues of previous investigations such as the meaning of the reflection coefficient and the instanton-like behaviour of the PRBB $\rightarrow$ POBB transition, and provides new, interesting results, for instance, the analysis of the full set of transition amplitudes and the role of the semiclassical approximation.

We have shown, through a concrete example, that the reduced phase space and the pathintegral approaches are extremely powerful techniques of quantization for a large class of string cosmology models. The two methods can be applied straightforwardly to any isotropic, spatially flat, model as long as the latter is characterized by scale factor duality invariance. In particular, the functional method may be very useful when the system cannot be integrated explicitly, i.e. when the Schrödinger equation (or, alternatively, the equivalent Wheeler-De Witt equation) cannot be solved exactly. Indeed, the calculation of the (semiclassical) transition amplitude between the PRBB and POBB phases in the weak-coupling regime is reduced to a simple evaluation of a definite integral by means of the residue theorem. No explicit solutions of the classical equations of motion nor exact wavefunctions are needed.

The path-integral method also makes clear a couple of other interesting features of quantum string cosmology models. First, we have proved that the instanton-like nature of the PRBB $\rightarrow$ POBB transition amplitude $[7,8]$ is just a consequence of the presence of the classical singularity in the strong-coupling regime. Indeed, the mere existence of the singularity implies that any semiclassical trajectory gives an $n$-instanton contribution to the PRBB $\rightarrow$ POBB transition amplitude. Secondly, we have clarified the role of the functional form of the dilaton potential in the transition process. We have mentioned that the dilaton potential may 'mimic' (at the quantum level) high-order corrections to the low-energy effective string theory action. The path-integral approach shows that the calculation of the semiclassical transition amplitude PRBB $\rightarrow$ POBB does not require the knowledge of the exact functional form of the dilaton potential. The semiclassical contribution to the transition amplitude is determined uniquely by the behaviour of the dilaton potential in the strong-coupling region. Thus for any dilaton potential whose asymptotic behaviour for $\Phi \rightarrow \infty$ is $V \sim \mathrm{e}^{a \Phi}$, where $a$ is a real positive parameter, the transition amplitude (in the semiclassical approximation) is known.

Let us conclude with an interesting speculation. The transition from the PRBB phase to the POBB phase can be described (phenomenologically) by an analytical continuation of the dilaton field to complex values. We have seen in section 3.2 that this analytical continuation can be interpreted in terms of a set of (complex) metrics with no well defined signature. This way of looking at an analytically continued solution as a quantum bridge connecting two classical hyperbolic spaces has a strong resemblance to the semiclassical Euclidean wormhole picture. Euclidean wormholes are classical instanton solutions of gravity-matter systems 
that (asymptotically) connect two manifolds [23]. They are usually interpreted as tunnelling between the two asymptotic configurations. In our case the transition from the PRBB phase to the POBB phase, at the semiclassical level, can be seen precisely as a wormhole-like effect. Our investigation provides the first example of the calculation of a wormhole-like tunnelling probability beyond the semiclassical level. This interpretation is very intriguing and supports the interesting suggestion that singularities in the classical domain of physical, hyperbolic solutions in gravity theories can be avoided by complex solutions joining two spaces, as happens in the case that we have discussed here.

\section{Acknowledgments}

We are very grateful to Maurizio Gasperini, Gabriele Veneziano and Alex Vilenkin for interesting discussions and useful suggestions. MC is supported by a Human Capital and Mobility grant of the European Union, contract no ERBFMRX-CT96-0012.

\section{References}

[1] Veneziano G 1991 Phys. Lett. B 265387

[2] Gasperini M and Veneziano G 1993 Astropart. Phys. 1317

[3] Gasperini M and Veneziano G 1993 Mod. Phys. Lett. A 83701 Gasperini M and Veneziano G 1994 Phys. Rev. D 502519

[4] See, e.g., Gasperini M, Maggiore M and Veneziano G 1997 Nucl. Phys. B 494315

[5] Brustein R and Veneziano G 1994 Phys. Lett. B 329429 Kaloper N, Madden R and Olive K A 1995 Nucl. Phys. 452677 Kaloper N, Madden R and Olive K A 1996 Phys. Lett. B 37134 Easther R, Maeda K and Wands D 1996 Phys. Rev. D 534247

[6] De Witt B S 1967 Phys. Rev. 1601113 Wheeler J A 1968 Battelle Rencontres ed C De Witt and J A Wheeler (New York: Benjamin-Cummings).

[7] Gasperini M and Veneziano G 1996 Gen. Rel. Grav. 28 1301-7

[8] Gasperini M, Maharana J and Veneziano G 1996 Nucl. Phys. B 472 349-60

[9] Maharana J, Mukherji S and Panda S 1997 Mod. Phys. Lett. A 12447

[10] Cavaglià M and de Alfaro V 1997 Gen. Rel. Grav. 29773

[11] Cavaglià M 1999 Int. J. Mod. Phys. D 8101

[12] Dirac P A M 1964 Lectures on Quantum Mechanics (New York: Belfer Graduate School of Science, Yeshiva University)

[13] Hanson A, Regge T and Teitelboim C 1976 Constrained Hamiltonian Systems (Rome: Accademia Nazionale dei Lincei)

[14] See, e.g., Henneaux M and Teitelboim C 1992 Quantization of Gauge Systems (Princeton, NJ: Princeton University Press)

[15] See, e.g., Metsaev R R and Tseytlin A A 1987 Nucl. Phys. B 293385

[16] Tseytlin A A 1991 Mod. Phys. Lett. A 61721

[17] Muller M 1990 Nucl. Phys. B 33737

Veneziano G 1991 Phys. Lett. B 265387

[18] Cavaglià M, de Alfaro V and Filippov A T 1995 Int. J. Mod. Phys. A 10611

[19] Hájíček P 1986 Phys. Rev. D 341040

[20] See, e.g., Abramowitz M and Stegun I A 1968 Handbook of Mathematical Functions (New York: Dover) pp 358-74

[21] See, e.g., Cavaglià M, de Alfaro V and Filippov A T 1996 Int. J. Mod. Phys. D 5227

[22] Itzykson C and Zuber J B 1980 Quantum Field Theory (Singapore: McGraw-Hill)

[23] See, e.g., Hawking S W 1990 Mod. Phys. Lett. A 5145

Hawking S W 1990 Mod. Phys. Lett. A 5453

Cavaglià M, de Alfaro V and de Felice F 1994 Phys. Rev. D 496493 and references therein 